\title{
TOMA DE DECISIONES ECONÓMICAS: EL APORTE COGNITIVO En la RUta de Simon, Allais y TVersky y Kahneman
}

\author{
ECONOMIC DECISION MAKING: A COGNITIVE CONTRIBUTION \\ ON THE PATH OF SIMON, ALLAIS, TVERSKY AND KAHNEMAN
}

\author{
Ricardo Pascale \\ Universidad de la Republica, Uruguay, \\ Facultad de Ciencias Económicas y de Administración \\ Gabriela Pascale \\ Universidad Católica del Uruguay, \\ Facultad de Psicología
}

\begin{abstract}
Resumen: Desde sus comienzos como ciencia, la economía integró a sus proposiciones a la psicología. Esto fue particularmente notorio en el tema de la toma de decisiones económicas. Posteriormente, la economía se recuesta para elaborar sus construcciones científicas en el maximizador y omnisciente homo economicus y así se separa de la psicología. Empero, la explicación de la realidad de los modelos neoclásicos se fue tornando cada vez más imperfecta, mostrando las teorías numerosas anomalías. Prominentes científicos en la vena cognitiva comienzan, primero exponiendo las limitaciones a las teorías prevalecientes para luego continuar construyendo modelos alternativos enraizados en la psicología cognitiva. Los seminales aportes cognitivos de Herbert Simon y Maurice Allais, trazarían el camino a dos académicos pioneros, los psicólogos cognitivos Amos Tversky y Daniel Kahneman, que darían forma a un nuevo modelo que incorpora los avances de la psicología cognitiva a los modelos de toma de decisiones. El trabajo revisa este paradigmático ejemplo de interdisciplinariedad científica entre economía y psicología cognitiva, en la búsqueda de mejores explicaciones al proceso de toma de decisiones.
\end{abstract}

Palabras claves: psicología cognitiva, teorías económicas, toma de decisiones.

\begin{abstract}
Economics, in its origins as a science integrated psychology in the building process of its propositions. The field of decision making was very notorious in economic research. Later on, economics takes some distance from psychology, and develop in the center of their scientific constructions, the maximizer and omniscient homo economicus. Nevertheless the explanatory power of the neoclassical models began to be clearly imperfect showing numerous anomalies. Prominent scientists in the cognitive vein began, first explaining the pitfalls of the prevalent theories and later building cognitive psychology-rooted alternative models. The seminal cognitive contributions by Herbert Simon and Maurice Allais, trace the path for two pioneers scholars, the cognitive psychologists Amos Tversky and Daniel Kahneman, who developed a model which incorporates the advances in cognitive psychology in decision making scientific constructions. The paper reviews this paradigmatic example of scientific interdisciplinary nature between economics and cognitive psychology, in the search for better explanations to the decision making process.
\end{abstract}

Key words: cognitive psychology, economic theories, decision making process.

Correspondencia: Prof. Mag. Gabriela Pascale. Universidad Católica del Uruguay. Facultad de Psicología. Departamento de Ciencias Cognitivas y de la Salud. 8 de octubre 2738. Montevideo. Uruguay. CP. 11600. Correo electrónico: gpascale@ucu.edu.uy. 


\section{A MODO DE INTRODUCCIÓN}

Este trabajo tiene como propósito exponer el proceso de renovado ensamble entre la psicología cognitiva y las decisiones económicas.

El mismo está concebido para poder ser transitado por una audiencia amplia, al enfocarse como una sustancial introducción al tema. Su uso, también puede extenderse como material introductorio al tópico a nivel de maestrías.

Desde los tiempos de Adam Smith, la economía se desarrolló integrando distintos aspectos de la psicología en sus construcciones científicas. Lo fue particularmente notorio en la teoría de las decisiones económicas, la teoría del consumidor y de los mercados.

Empero, al promediar la primera mitad del siglo XX, ambas disciplinas en cierta forma se separan y continúan, cada una, su camino en sus avances científicos.

Durante el Siglo XX la Psicología emergió como un campo de estudio distinto de la Filosofía y de la Fisiología. Desde el nacimiento de la llamada Psicología Científica se han producido enormes avances en el camino de asentar las posiciones epistemológicas posteriores como fueron la psicología hasta entonces dominada por el paradigma conductista del Estimulo-Respuesta y la Psicología Cognitiva.

La economía, por su parte se formalizaba en modelos matemáticos alejándose de varias disciplinas de las que tomaba conocimiento, tal el caso de la psicología, la historia o la antropología.

Los avances científicos, en el campo de la economía, sin embargo, -en la segunda mitad del siglo XX-, comienzan a cuestionarse en particular varios modelos económicos, al advertirse numerosas anomalías en las predicciones de los mismos. Este fenómeno fue muy notorio en los modelos vinculados a la toma de decisiones económicas. Comienza de esta forma, en la segunda mitad del siglo pasado a construirse un camino hacia la "reunificación" de ambas disciplinas en la teoría de las decisiones económicas (Camerer, 1999). Este proceso se acentúa a partir de los años setenta del Siglo XX, donde un grupo de connotados psicólogos cognitivos, hacen aportes que mejoran seriamente las predicciones que surgían de los modelos económicos precedentes.

El trabajo está estructurado de la siguiente forma: En la sección segunda se repasa este arco evolutivo de la relación de ambas discipli- nas, buscando dejar en claro "el problema" a abordar por el mismo.

La sección tercera da cuenta de tres aspectos fundamentales para facilitar la comprensión del problema bajo exposición. Estos son: la racionalidad subyacente en la economía neoclásica, una aproximación a las ciencias cognitivas y, finalmente se centra en la psicología cognitiva.

La cuarta sección está dedicada a presentar las principales paradojas y anomalías, que se advierten entre las predicciones de la teoría económica neoclásica y la realidad, donde la psicología cognitiva juega un rol central.

La quinta sección se centra en la "Prospect Theory" desarrollada por los psicólogos cognitivos Amos Tversky y Daniel Kahneman, que es un ejemplo paradigmático de la inserción reciente y exitosa de esta disciplina en la teoría de la toma de decisiones económicas.

Por último el trabajo concluye con las consideraciones finales.

\section{2. "POR QUIEN DOBLAN LAS CAMPANAS"}

Tradicionalmente, desde los albores de la "moderna" economía, la interrelación entre psicología y economía, como expresamos, han sido muy grandes.

El eminente economista Alfred Marshall (1920), en su significativa obra "Principios de Economía" señala que:

"La economía es una ciencia psicológica. La Economía Política o Economía es el estudio de los seres humanos en la vida ordinaria de los negocios, examina esa parte de la acción individual y social que está más estrechamente conectada con los logros y con el uso de los requisitos materiales para el bienestar.

Entonces es, por un lado, el estudio de la riqueza, y por el otro, un lado más importante, una parte del estudio de hombre. Fue modelada para el carácter de los hombres en su trabajo del día a día y los recursos que ellos procuran sin otra influencia a no ser la religiosa.

En su actual desarrollo, sin embargo, la ciencia económica ha focalizado solamente en un aspecto del carácter del Hombre, su razón, y particularmente en la aplicación de esa razón a los problemas de asignación de recursos en la fase de escasez. 
Todavía, las modernas definiciones de las ciencias económicas, ya sea expresada en términos de asignar recursos escasos o en términos de toma de decisiones racionales, dejan fuera un vasto dominio para conquistar y establecerse. En años recientes ha habido exploraciones considerables efectuadas por economistas, de partes de sus dominios, las cuales tradicionalmente eran pensadas como pertenecientes a otras disciplinas como la ciencia política, la sociología y la psicología."

Desde el momento en que la economía es una ciencia que se ocupa de cómo los individuos y las firmas e instituciones asignan los recursos, la psicología cognitiva estudia los procesos mediante los cuales estos individuos se representan la realidad, estando estos en la base de la información fundamental de la que se nutren los agentes económicos para la toma de decisiones.

Los desarrollos de la economía, desde los tiempos de Adam Smith hasta promediar la primera mitad del Siglo XX, los trabajos de economistas de singular talla como Keynes, Marshall, Fisher o Hayek, están impregnados de insumos, percepciones, apreciaciones y presentaciones psicológicas. Si bien en economía el libro más conocido de Adam Smith es "La Riqueza de las Naciones", (1776), años antes, en 1759, escribió "La Teoría de los Sentimientos Morales", en donde examinó estos sentimientos los cuales priman en la propia naturaleza de los individuos.

Vernon L. Smith ${ }^{1}$ (2002) señala al respecto:

"Contrariamente a la opinión común, en la visión de Smith (Adam) cada individuo define y persigue su propio interés a su modo, están mal caracterizados por la metáfora del "Homo Economicus". Este poco depurado conocimiento por parte de los estudios les ha impedido tomar la proposición clave del filósofo escocés"

Las ideas de su primer libro permanecen, por lo común, ignoradas por los economistas.

Dos factores han sido los principales en ese distanciamiento de la economía con respecto de la psicología, las que comienzan a avanzar por senderos distintos.

El primero de estos factores es el énfasis que en economía se le empieza a dar al instrumental matemático, apoyándose principalmente en conceptos de la física. En economía se habla de estática, dinámica, multiplicador, aceleración, tasa. Estos primeros economistas matemáticos entre los cuales se encuentran R. Frisch, P.A. Samuelson. J. Tinbergen, K. Arrow, G. Debreu, T. Koopmans, científicamente hablando provienen de la física. La explicación de los fenómenos económicos para el economista, pasa de esta forma a basarse en teorías con el sustento de un cuerpo de herramientas matemáticas y teoremas.

El segundo factor, deriva de que buena parte de los economistas toman una variación de la lógica positivista, impulsada principalmente por el distinguido economista de la Universidad de Chicago, Milton Friedman. Señala Friedman ${ }^{2}$

"Completo "realismo" es claramente no obtenible y, la pregunta de si una teoría es "suficientemente" realista debe ser vista en términos de sus predicciones son suficientemente buenas para el propósito que se tiene en mano".

Y agrega más adelante:

"Hipótesis verdaderamente importantes tendrán supuestos que son brutalmente inapropiadas representaciones descriptivas de la realidad y, en general, cuanto más significativa la teoría, más irrealistas los supuestos".

Refiriéndose a estas anotaciones Simon (1963) establece:

"Permítaseme proponer un principio metodológico para reemplazar el principio de la irrealidad de Friedman. Me gustaría llamarlo continuidad de aproximaciones. Consiste en que si las condiciones del mundo real se aproximan suficientemente bien a los supuestos de un tipo ideal, las derivaciones en ese supuesto serán aproximadamente co-

\footnotetext{
1 Vernon L. Smith, recibió el Premio Nobel en Economía en 2002 “Por haber establecido experimentos de laboratorio como una herramienta en el análisis empírico económico, especialmente en el estudio de las alternativas de los mecanismos de mercado".

${ }^{2}$ Milton Friedman, recibió el Premio Nobel de Economía en 1976 "Por sus logros en los campos del análisis del consumo, historia y teoría monetaria y por su demostración acerca de la complejidad de la política de estabilización."
} 
rrectas. La irrealidad de las premisas no es una virtud de una teoría científica, es un mal necesario -una concesión a la capacidad finita de cómputo del científico- que es tolerable por el principio de la continuidad de la aproximación".

Las críticas a la irrealidad también vinieron de Paul A. Samuelson (1963) que estableció que si bien el irrealismo es, a veces, un mal necesario, lo llamó "un demérito para cualquier teoría".

Estas críticas metodológicas, no menguaron el camino divergente que tomó la economía de otras áreas, entre ellas la psicología.

De esta forma la psicología y la economía neoclásica transitaron y avanzaron sus conocimientos por varias décadas del siglo pasado por caminos bifurcados. La psicología, pone el acento en la comprensión de la naturaleza de los elementos de las decisiones, del modo en que ellas se establecen y son modificadas en la experiencia, del modo en las cuales se determinan los valores. Asimismo, la visión psicológica del proceso decisional está influenciada por la idea de contexto cambiante e influenciado de interacciones de percepciones, motivos y emociones.

La economía neoclásica, basándose en que lo importante está en el "with the purpose on hand", el decididor se comporta como si la información fuera elaborada para formar percepciones y creencias utilizando rigurosos principios estadísticos.

De esta forma el hombre que maneja la economía neoclásica es como señala Mc Fadden ${ }^{3}$ (1995) el "hombre de Chicago" (en esta Universidad aparecen además de Friedman, otros distinguidos economistas que acompañan esa posición como Becker (1993) y Lucas (1987)). El "hombre de Chicago" es un hombre perfectamente racional, omnisciente y que busca su propio interés. Es, por tanto, un hombre maximizador, optimizador. La economía sienta una posición epistemológica bien definida, que para poder transitar, se recuesta en el ceteris paribus.

Por este sendero, de fuerte impronta matemática, la economía efectuó empero avances significativos. Asimismo, en esas décadas fue criticada por cientistas sociales que advirtieron de la utilización de la perfecta racionalidad. Los economistas, por su parte, defendían sus posiciones, señalando que sus modelos, en todo caso, aportaban aproximaciones útiles.

Como hemos señalado estos tiempos de distanciamiento entre psicología y economía, comenzarían a cambiar a lo que se ha dado en llamar una "reunificación" de ambas (Camerer, 1999), a partir de los años 50 del siglo pasado. La figura, que comienza este proceso fue Herbert Simon (1947, 1957, 1969, 1971, 1972, $1971,1991)$ quien acuñó el término que hoy se conoce como "racionalidad acotada" (bounded rationality). Simon, fue además de economista, psicólogo, brillante matemático y cientista de las tecnologías de la información y pionero de los avances de la inteligencia artificial. En su brillante carrera, cosechó todo tipo de honores incluyendo el Premio Nobel de Economía en $1978{ }^{4}$ y The Gold Medal de la Fundación Americana de Psicología (APA) en 1988 así como también recibe el galardón A.M Turing en ciencias computacionales. Su publicación conjuntamente con Alan Newell y J. C Shaw sentaron uno de los primeros esfuerzos por encontrar modelos decionales que superen los supuestos del hombre económico racional. Sus demostraciones matemáticas fueron llevadas a cabo por programas con lenguaje computacional como fueron el Logic Theory Machine y el General Problem Solving. Si bien estos postulados en ese momento no prosperaron ampliamente, no se puede dejar pasar inadvertida la influencia que han tenido en algunas áreas de debate sobre el pensamiento humano y la resolución de problemas. Tal es el caso de Neisser (1967) quien reconoce que la metáfora de la mente humana con el ordenador se encontraba en estos primeros trabajos de Simon.

Sus teorías, sobre la base de "racionalidad acotada" desarrollan la forma en la que los individuos toman las decisiones a través de algoritmos que tienen incorporados mecanismos de los cuales se ocupa la psicología cognitiva. La toma de decisiones resulta el output en términos de conductas de cierta información que es procesada (pensamiento) por las variables mediadoras al interior del sistema.

\footnotetext{
${ }^{3}$ Daniel Mc Fadden, distinguido economista de la Universidad de Berkeley, recibe en el año 2000 el Premio Nobel de Economía "por sus desarrollos en la teoría y los métodos para analizar elecciones discretas".

${ }^{4}$ Herbert Simon recibe el Premio Nobel en Economía en 1978 "Por sus amplias contribuciones a la comprensión del proceso de toma de decisiones, en especial en organizaciones económicas."
} 
La teoría propuesta busca dar respuesta a la pregunta de, ¿cómo los seres humanos adoptan las decisiones económicas, en la realidad, en el seno de las organizaciones?

La teoría se basa en tres aspectos principales; a) la relación de empleo, b) el equilibrio organizacional y c) los mecanismos de la racionalidad acotada.

Con respecto a la relación de empleo, Simon establece que una de las características fundamentales de las economías modernas está basada en que en la mayoría de los casos un individuo no produce un producto para la venta, sino que el trabajo está dado por relaciones de empleo en una empresa o en una organización y que supone una relación jerárquica basada en la autoridad con el empleador. La forma en que ese individuo organice su experiencia va a determinar el reconocimiento de este aspecto el cual va a condicionar al menos hasta ciertos límites a la del empleador.

En cuanto al equilibrio organizacional, desarrolla el concepto en el marco de una teoría motivacional, del balance entre las aspiraciones de los participantes del sistema (inversores, empleados, clientes, proveedores, etc.). Las condiciones de supervivencia de una compañía se transforman en la de resultados económicos positivos, pero como dice Simon:

"mientras la teoría tradicional de la firma supone que los beneficios van hacia un sector en particular, los propietarios, la teoría de la organización supone una distribución más simétrica y no predice como se distribuirá".

Los mecanismos de racionalidad acotada, se basan en dos pilares básicos: a) búsqueda de alternativas y b) satisfacción. Al contrario de lo que sostiene la teoría económica tradicional, en el sentido que se buscará la mejor alternativa, es decir, la que maximizará el beneficio, Simon sostiene una posición diferente. Entiende que el individuo no conoce todas las alternativas, esto es, cuenta con una información limitada, es decir hay una escasez de data al tiempo que de programas que utilicen la información. El agente económico busca alternativas y, cuando encuentra la que se aviene a su nivel de apreciación, desiste de la búsqueda de otras alternativas y escoge esa. Esta forma de buscar alternativas, Simon la denomina como un modelo de selección de satisfacción. De esta forma, entiende que los agentes económicos satisfacen más que maximizan.

En suma, el modelo neoclásico se desarrolla en términos que los agentes económicos conocen todas las alternativas y que las evalúan correctamente $y$, por tanto, deciden en términos óptimos, maximizando.

Simon, en cambio sostiene que la tarea es "reemplazar el modelo clásico con uno que describiera como las decisiones pueden ser (y probablemente actualmente son) hechas cuando las alternativas de búsquedas deben ser miradas y las consecuencias de cada una de ellas son imperfectamente conocidas" y propone sus aportes de racionalidad acotada a estos efectos, en donde sostiene que los agentes económicos satisfacen en lugar de optimizar.

En el campo de la racionalidad acotada, aparece clave el método utilizado que se asienta en la observación experimental directa de los fenómenos psicológicos a nivel individual, en donde juegan un papel decisivo el razonamiento inductivo y los mecanismos y esfuerzos por sortear obstáculos en la búsqueda de resolución de problemas. Estos serían algunos de los elementos centrales del complejo del pensamiento humano que guía las decisiones económicas.

Simon, para poder explicar la actividad cognitiva humana considera que es fundamental elaborar un modelo de representación mental adecuado que dé cuenta del contexto decisional de la mejor manera posible. Las decisiones según Simon no solo son fruto de las capacidades cognitivas limitadas sino también de las representaciones que el decididor en base a su percepción se haga de entorno, introduciendo conceptos que luego en su obra desarrollará como es el caso del aprendizaje social. En el esfuerzo por entender las variables medidoras en la que se asientan ciertas decisiones humanas relega los modelos de economía matemática y la econometría para enfatizar en otros estudios de base empírica. Así es que trabaja observando el cálculo decisional en los jugadores de ajedrez y se va introduciendo en un modelo cognitivo. Empero la vasta obra de Simon abarcó la inteligencia Artificial en donde por encima de estudios de base empírica incorpora refinado instrumental matemático.

La teoría de Simon no parece fácil de conciliar con la corriente más importante en economía, que tiene un claro sesgo matemático. Sus contribuciones serían de tal entidad que Mc Fadden (1995) diría luego: 
"el hombre de Chicago es una especia en extinción”.

En efecto, son numerosas las anomalías -los hechos u observaciones que son inconsistentes con las teorías- comenzadas a exponer por Simon y continuadas por muchos académicos.

Encontrar una anomalía puede llegar a considerarse un episodio curioso o anecdótico. Cuando las anomalías son muy numerosas, están suponiendo nuevos caminos. Kuhn (1962) comenta que:

"el descubrimiento comienza al advertir anomalías, por ej. con el reconocimiento de que la naturaleza ha violentado el paradigma que prevalece en la ciencia normal". ${ }^{5}$

Los psicólogos cognitivos comienzan estudiando juicios y decisiones económicas. Toman como base, la maximización de las utilidades y los principios probabilísticas bayesianos como objetivo y, utilizan conformidades o desviaciones con respecto a los objetivos que teorizan sobre los mecanismos cognitivos que las producen. Entre estos psicólogos se destacan Ward Edwards y luego Amos Tversky, Daniel Kahneman, Baruch Fischhoff, Paul Slovic. Cabe destacar que en el caso de Mattew Rabin y Richard Thaler son economistas que han trabajado estrechamente con psicologos.

Los resultados de estas investigaciones coinciden en que principios de la psicología cognitiva podían ser expresados en términos formales. De esta manera, este modo de incorporar la psicología provee un medio para modelizar la racionalidad acotada en términos más "standard" para la economía, que la aproximación inicial efectuada por Simon. Empero las proposiciones de este ultimo serían claves para abrirle el camino al campo cognitivo en amplias áreas de la econimina entre la que se encuentra las finanzas comportamentales.

Un excelente ejemplo de cómo la psicología cognitiva mejora las predicciones económicas se tiene en la "Prospect Theory" que desarrollaron Tversky y Kahneman ${ }^{6}(1979,1987,1992)$.

\section{FUNDAMENTOS}

\subsection{La racionalidad en la teoría económi- ca neoclásica.}

3.1.1. Status epistemológico neoclásico

La "cintura protectiva", á la Lakatos de la economía neoclásica se componía sobre los años cincuenta del siglo XX entre otros aspectos por una perfecta racionalidad individual y colectiva, donde el cálculo era el elemento dominante y, se suponía, por tanto la optimización de las elecciones, así como por su propio interés. Lionel Robbins (1932) expone la desde entonces ampliamente recibida definición de economía, que resume el status original que se quiere señalar. En su famoso ensayo establece que la "economía es la ciencia que estudia el comportamiento humano como una relación entre fines dados y medios escasos que tienen usos alternativos".

En estas ideas, además de considerar a la economía una "ciencia deductiva", es posible calcular el comportamiento de los individuos, toda vez que se conozcan los fines y los medios, así como las preferencias.

La aproximación paradigmática de Robbins ubica a la economía, como una disciplina completamente despreocupada de comprender los elementos de la psicología de la elección.

En ese momento, era poco probable señalar que la economía podría ser también una ciencia experimental, donde la psicología de la elección juegue un papel significativo.

En aquellos tiempos, al decir de McFadden ${ }^{7}$ (1995), el "hombre de Chicago" es el que predomina y queda conformado con un modelo convencional de racionalidad, tanto sea de percepción, como de preferencias y de los procesos.

Racionalidad de percepción, implica que quien toma la decisión se comporta como si fuese elaborada para formar percepciones y creencias a través del uso de rigurosos principios es-

\footnotetext{
${ }^{5}$ En la posición de Kuhn, además de la presencia de anomalías, no sería factible desplazar la teoría anterior sino se cuenta con una nueva teoría.

${ }^{6}$ Daniel Kahneman recibió el Premio Nobel en Economía en 2002 (siendo así el primer psicólogo en obtenerlo) "Por haber integrado profundizaciones de la investigación psicológica en la ciencia económica, especialmente en lo concerniente al juicio humano y la toma de decisiones en condiciones de incertidumbre". Amos Tversky, había fallecido antes, sino sin duda, como lo dejó entrever la Real Academia Sueca de Ciencias también lo habría recibido.

${ }^{7}$ McFadden recibe el premio Nobel de Economía en el año 2000, "por sus desarrollos en la teoría y los métodos para analizar elecciones discretas".
} 
tadísticos bayesianos. La racionalidad de preferencias nos habla de que ellas aparecen primitivas, coherentes e inmutables. Por último, la racionalidad del proceso, da por sentado que los procesos cognitivos son simplemente maximizadores de las preferencias, informaciones y vínculos de mercado.

El "hombre de Chicago" está conectado unidireccionalmente desde los flujos de las percepciones y de los gustos a la tarea cognitiva de la maximización de las preferencias. Muchos autores se preguntan (Mc Fadden, 1995):

"Porqué cuando los economistas son puestos de frente a la evidencia comportamental adversa a ese modelo, tergiversan, numerosas excusas y luego continúan a hacer lo que estaban haciendo?" (Mc Fadden, 1995)

Creemos que la explicación va más allá del "hombre de Chicago" y del farol bajo el cual muchos economistas buscan la verdad. ${ }^{8}$

En realidad "el hombre de Chicago", ha sido de gran utilidad para hacer avanzar el conocimiento de amplios sectores de la economía, como el análisis de la demanda, el costo beneficio, así como arbitrajes en los mercados financieros.

Las ciencias avanzan, a veces, con supuestos simplificadores. El como si, y que sea útil para los propósitos que se tienen a la mano, permitió muchos avances.

Hoy, existe una importante evidencia empírica en contra a una interpretación literal del "hombre de Chicago", que en definitiva es el Homo Economicus como modelo universal para explicar y predecir las elecciones y la toma de decisiones. Este Homo Economicus, va tendiendo a sustituirse por el Homo Sapiens (Thaler, 2000).

Un lector superficial de Simon, de Kahneman o de Tversky puede confundir la insuficiencia de la racionalidad perfecta con la idea de que el hombre es irracional. El hombre se supone que opera racionalmente, pero en el contexto de un complejo proceso cognitivo dentro de lo que se puede caracterizar como racionalidad procedimental.
Otra creencia, que es preciso aclarar es que la psicología cognitiva, no tiene interés en destruir los enormes avances que realizó la teoría económica. Realiza, empero aportes sustanciales para mejor informar a los economistas e introduce en sus modelos un componente cognitivo con el objeto de explicar más adecuadamente la realidad del decicidor.

\subsubsection{La utilidad esperada}

La mayor precisión formal de esta aproximación neoclásica, se obtiene con el famoso texto de John von Neumann y Oskar Morgenstern (1944), que extienden el trabajo que doscientos años antes había realizado Daniel Bernoulli (1738).

El tema lo centraron en la demostración de la insuficiencia del valor monetario esperado (VME) como criterio para decisiones riesgosas y llegaba hasta la teoría de la maximización de la utilidad esperada (MUE).

En el caso del VME, en situaciones de elección se suponía que el objetivo era maximizar el rendimiento esperado en dinero.

Supóngase, considerando un ejemplo sencillo y tomando ciertos supuestos, que tenemos que decidir entre dos opciones. Un negocio A que tiene tres eventualidades, que son $\$ 6.000$, $\$ 4.000$ o $\$ 1.000$ de ganancia con probabilidades de $0.3,0.4$ y 0.3 , respectivamente. En este caso el valor monetario esperado es $\$ 3.700^{9}$

El negocio $B$ tiene las eventualidades de perder 10.000 o de ganar $\$ 20.000$ o $\$ 7.000$, con probabilidades respectivas de $0.5,0.4$ y 0.1 . El valor monetario esperado de $B$ también nos da $3.700^{10}$. Según este criterio ${ }^{11}$, matemáticamente muy utilizado durante mucho tiempo, sería indiferente elegir una opción u otra. Sin embargo, en la realidad, los sujetos se inclinan por $\mathrm{A}$ o por $\mathrm{B}$, más allá de que tengan el mismo VME. Se introducen así las preferencias subjetivas ante el riesgo.

Esta insuficiencia del valor monetario esperado fue expuesta por Bernoulli en 1738 en la Academia de Ciencias de San Petersburgo. Él distingue entre la suma dineraria (pretium) y la utilidad que ella le reporta al sujeto (emolumentum).

\footnotetext{
${ }^{8}$ Esta referencia al farol corresponde a un conocido cuento sobre economistas: "Una noche un policía ve a un economista afanado en buscar algo bajo el farol de una esquina y le pregunta que está buscando". El economista le responde de haber perdido la llave de su casa, en el frente de ella. El policía le pregunta porque está buscándola entonces bajo el farol de esta esquina". El economista responde: "Porque aquí tengo luz y veo mejor".

$9-6.000 \times 0.3+4.000 \times 0.4+1.000 \times 0.3=3.700$

$1010.000 \times 0 .+20.000 \times 0.4+7.000 \times 0.1=3.700$

${ }^{11}$ En estadística para determinar el valor esperable de una variable aleatorio discreta se debe multiplicar cada posible valor de la variable por la probabilidad y sumar sus resultados.
} 
Y allí plantea la discusión si es erróneo o no que se venda en 9.000 ducados un billete de lotería que tenía iguales probabilidades de obtener cero o 20.000 ducados. (el valor monetario esperado es 10.000 ducados, o sea $0.5 \times 0$ $+0.5 \times 20.000$ ).

Expone, lo que hoy se conoce como la "Paradoja de San Petersburgo" de la siguiente forma:

Una vez un pobre hombre obtuvo un ticket de lotería que le rendiría con igual probabilidad nada ó 20.000 ducados ¿Habría este hombre evaluado su chance de ganar 10.000 ducados? ¿Habría sido mal aconsejado en vender su ticket por 9.000 ducados?

Me parecía que la respuesta era negativa. Por otro lado, estoy inclinado a creer que un hombre rico será mal aconsejado si se negara a comprar el ticket de lotería por 9.000 ducados. Si no me equivoco, entonces me parece claro que no todos los hombres pueden usar la misma regla para evaluar las apuestas. La regla establecida con anterioridad debe, por lo tanto, ser descartada. Cualquiera que considere el problema con perspicacia e interés averiguará que el concepto de valor que usamos en esta regla debe ser definido de forma que interprete cabalmente un procedimiento universalmente aceptado sin reservas. Para esto, la determinación del valor de un artículo depende solamente de sí mismo, y es igual para cualquiera; la utilidad, sin embargo, depende de las características particulares de la persona que la estima. No hay duda de que una ganancia de 1.000 ducados es más significativa para un pobre que para un hombre rico, aún cuando el monto de la ganancia es el mismo.

El concepto del valor monetario esperado dejó paso a otro más complejo, el de la utilidad esperada. Esta utilidad tiene relación con las actitudes de los individuos ante el riesgo.

Esta utilidad, medida no en términos de ganancia sino de satisfacción, que incluye las preferencias subjetivas ante situaciones inciertas, fue un paso decisivo para considerar superado el valor monetario esperado y dar paso a la utilidad esperada.

Transcurrió mucho tiempo hasta que John von Neumann y Oskar Morgenstern
(VN -M) en "Theory of Games and Economic Behavior" de 1947, establecieron que la utilidad es un número que utiliza quien debe adoptar una elección de las retribuciones en condiciones de incertidumbre. El concepto de VN-M es, en definitiva, un intento de crear una teoría del comportamiento racional, aunque se mantuvo en la vena neoclásica.

La idea central de la teoría es que una apuesta realizada con iguales oportunidades no es necesariamente equitativa, salvo cuando implica para el jugador ventajas y desventajas iguales. La simetría de ganancias y pérdidas en moneda en modo alguno es la simetría de utilidades y desutilidades económicas. En el decir de Marschak, "una bolsa llena no es tan buena, como no es tan mala una bolsa vacía".

VN-M, elaboran entonces una función de utilidad, la transforman en mensurable. En esto la distinguen de la utilidad ordinal de J. Hicks, y R. Allen (1939). Pero aunque es mensurable, la distinguen de la utilidad cardinal de Alfred Marshall, pues para él es una cantidad psicológica para medir el placer y el dolor, el concepto VN-M es un índice numérico para evaluar situaciones inciertas.

En general, se supone que es un soslayador de riesgo quien cede valor monetario esperado. Ese es, en el caso del ejemplo de Bernoulli, el de quien vendía su billete en 9.000 ducados, cuando 10.000 ducados era el valor monetario esperado.

La teoría de la utilidad esperada se asienta en varios axiomas tales como: el ordenamiento que involucra dos principios, el de asimetría (el sujeto prefiere una naranja a una ciruela) y transitividad (si el sujeto prefiere una naranja a una ciruela y, una ciruela a una pera, preferirá una naranja a una pera); el axioma de la continuidad (de comodidad operativa matemática) y el de independencia (si se prefiere una naranja a una ciruela, preferirá una lotería en la cual tiene una probabilidad de 0,4 de ganar una naranja y de 0,6 de ganar un traje, que otra que tenga 0,4 de ganar una ciruela y 0,6 de ganar un traje). A partir de estos axiomas, la función de utilidad se basa en algunas propiedades como:

- debe ser el resultado A preferible al $\mathrm{B}$, la utilidad de $A$ es mayor que $B$, lo cual se expresa como:

$$
U(A)>U(B)
$$


- si una persona se encuentra en una situación $Y$ que le representa una compensación A con la probabilidad p y una compensación $B$ con probabilidad 1-p, la utilidad de $Y$ es igual a:

$$
U(Y)=p U(A)+(1-p) U(B)
$$

A partir de estas propiedades se puede construir la curva de la función de utilidad, para lo cual se seguirá un ejemplo:

Se supone que existen probabilidades $p=0,5$ de ganar en una lotería $0, y(1-p)=0,5$ de ganar 100.000 .

Se le asigna a 0 un índice de utilidad 0 , y a 100.000 un índice 1. La elección de estos índices es arbitraria; lo importante es identificar la escala. Existen múltiples ejemplos de diferentes escalas para representar el mismo fenómeno. De esta forma, para la medición de la temperatura, la escala que trabaja con grados centígrados ha tomado como grado 0 el de congelación del agua, y 100 el de ebullición. La escala Fahrenheit tiene para estos dos mismos fenómenos -32 y 212 , respectivamente- dos escalas distintas que representan lo mismo. Lo importante es pues, como se decía, identificar la escala.

En este ejemplo se han tomado 0 y 1 , lo mismo que se podrían haber tomado 10 y 100 , ó 50 y 900 .

Continuando con preguntas se pueden seguir obteniendo valores de las curvas de utilidad del individuo. Por ejemplo, se le puede preguntar si las probabilidades fueran $p=0,6$ y $p=0,4$, por qué monto cedería el billete. Suponiendo que conteste 50.000 se tiene que:

$$
\begin{aligned}
& U(50.000)=0,6 U(100.000)+0,4 U(0) \\
& \text { o sea: } \\
& U(50.000)=0,6 \times 1+0,4 \times 0=0,6
\end{aligned}
$$

Se le pregunta luego al individuo por qué cantidad cierta está dispuesto a cambiar el billete.

Suponiendo que contesta 35.000 , se tiene que:

$$
\begin{aligned}
& U(35.000)=0,5 U(100.000)+0,5 U(0) \\
& \text { o sea: } \\
& U(35.000)=0,5 \times 1+0,5 \times 0=0,5
\end{aligned}
$$

Y así sucesivamente se podría ir formando la curva.
Un individuo averso al riesgo tiene una función de utilidad cóncava. Arrow (1965) y Pratt (1964) desarrollan una medida de absoluta aversión al riesgo, que viene definida como:

$$
A A R=\frac{-W(U)^{\prime \prime}}{W(U)^{\prime}}
$$

Esto es, la inversa del cociente de la derivada segunda y de la derivada primera de la función de utilidad.

Hacia esos años, empero, iban apareciendo críticas a la teoría de la utilidad esperada. Dos de ellas serían fundamentales. Una provendría de Europa y, tendría como exponente principal a Maurice Allais que demuestra que los axiomas en que se basa la teoría de la utilidad esperada, son sistemáticamente violados en las decisiones de los individuos y, la otra desde Estados Unidos, cuya figura más importante sería Herbert Simon, que se ocupa de la toma de decisiones en organizaciones. Las mismas se exponen sumariamente en las secciones que siguen.

\subsection{Ciencias Cognitivas}

Las ciencias cognitivas son un conjunto de disciplinas conformado por la Antropología, Inteligencia Artificial, Psicología Cognitiva, Filosofía de la mente, Lingüística, y la Neurociencia que comenzaron a emerger en la segunda mitad del siglo XX. Existe un consenso que la fecha de nacimiento de las Ciencias Cognitivas fue entre el 10 y el 12 de setiembre de 1956 en un Simposio sobre la Teoría de la Información realizado en el Instituto de Tecnología de Massachussets. Durante el mismo, grandes figuras provenientes de distintas disciplinas confluyeron exponiendo sus germinales trabajos e investigaciones. Se destacó la presentación de Hebert Simon y Allan Newell sobre "La maquina de la Teoría lógica" y de Noam Chomksy sobre los "Tres modelos del Lenguaje". En los años sesenta se despliega una enorme cantidad de producción en esta nueva área del conocimiento, con la aparición de numerosísimos escritos, artículos y centros de investigación dedicados a la misma.

Las ciencias cognitivas tienen como tarea común el comprender la mente humana desde los distintos ámbitos de desarrollo. Si bien se basan en diversos métodos utilizan la lógica de la 
representación, en la cual la mente puede ser entendida como un sistema de símbolos análogo al computador. Es decir, como se representa la información en la mente.

Sus aportes han sido de tal influencia que puede decirse que de hecho ninguna disciplina que estudie el quehacer humano puede quedar ajena a las ciencias cognitivas. Gardner (1988) la define como:

"Empeño contemporáneo de base empírica por responder a interrogantes epistemológicos de antigua data, en particular los vinculados a la naturaleza del conocimiento, sus elementos componentes, sus fuentes, evolución, y difusión"

\subsection{Psicología Cognitiva}

Las ciencias cognitivas le permiten a la Psicología Cognitiva liberarse del paradigma epistemológico dominante de la psicología estadounidense (el del E-R). El nacimiento de la misma surge como un movimiento en reacción por intentar abordar el estudio del ser humano desde otro ámbito: la mente.

Como expresa Fernández Álvarez (1992):

"La Psicología cognitiva vuelve a plantear la necesidad de adentrarnos en la intimidad del sujeto, en la privacidad de la mente, en los espacios secretos de la subjetividad para tratar de responder del mejor modo posible a la pregunta de cómo tienen lugar el conocimiento ¿cómo llegamos a conocer lo que conocemos? (...)

Un grupo de connotados psicólogos fueron pioneros en la construcción de esta corriente de pensamiento. Entre ellos pueden mencionarse los estudios de George Miller, Donald Broadbent, Colin Cherry y los estudios sobre las capacidades limitadas para el procesamiento de la información, Jerome Bruner sobre el planteamiento de estrategias para el desempeño de tareas, y Noam Chomsky con las críticas conductistas a las aproximaciones lingüísticas.

\section{ANOMALIAS Y PARADOJAS}

\subsection{Crítica de Allais}

En 1952, en un congreso en París, Allais presentó la crítica a la escuela americana y en particular a la posición de Milton Friedman, demostrando que, con un método experimental los individuos puestos frente a elecciones alternativas, violaban sistemáticamente los comporta- mientos previstos por la teoría de la utilidad esperada.

Estos hallazgos son hoy conocidos como la "paradoja de Allais". Su experimento pivotea sobre los axiomas de la utilidad esperada, y son ellos, los que son violados en sus experimentos por parte de los sujetos. Difundidos posteriormente los trabajos de Allais (1953), expone su experimento efectuado con personas a quienes le presentó dos elecciones hipotéticas.

La primera elección era entre $A$ y $B$, definida de la siguiente forma:

- Alternativa A: certeza de recibir 100 millones de francos

- Alternativa B:

- probabilidad 0,10 de recibir 500 millones

- probabilidad 0,89 de recibir 100 millones

- probabilidad 0,01 de no recibir nada.

La segunda elección, Allais la plantó de la siguiente manera:

- Alternativa C:

- probabilidad 0,11 de recibir 100 millones

- probabilidad 0,89 de no recibir nada

- Alternativa D:

- probabilidad 0,10 de recibir 500 millones

- probabilidad 0,9 de no recibir nada.

Siguiendo los axiomas de la utilidad esperada, un individuo que prefiera $A$ con respecto a $\mathrm{B}$, debe asimismo preferir $\mathrm{C}$ a $\mathrm{D}$. La mayoría de los sujetos que participaron en el experimento, prefirieron, contrariamente a lo postulado por la utilidad esperada a $\mathrm{D}$ en lugar de $\mathrm{C}$.

Esta violación de la utilidad esperada se puede expresar sabiendo que si $A>B$

$$
\begin{aligned}
& U(100)>0,10 \cup(500)+0,89 U(100)+ \\
& 0,01 U(0), \\
& \text { o sea } \\
& 0,11 U(100)>0,10 U(500)+0,01 U(0)
\end{aligned}
$$

para que $D$ sea preferido a $C$ si

$$
\begin{aligned}
& 0,11 U(100)+0,89 U(0)<0,1 U(500)+ \\
& 0,90 U(0) \\
& 0,11 U(100)<0,10 U(500)+0,01 U(0)
\end{aligned}
$$

El experimento fue repetido en numerosas oportunidades por diversos investigadores con 
resultados similares a los encontrados por Allais. Desafortunadamente, sus aportes no fueron oportunamente valorados en su justa dimensión por el mundo académico. Arrow ${ }^{12}$ ha buscado una explicación a esta situación en base al ambiente académico más reducido, donde silenciosamente trabajaba Allais $^{13}$, y se hubieran ganado, señala el reconocido economista estadounidense, treinta años en llegar a los adelantos que posteriormente se dieron es esta área.

\subsection{Heurística, sesgos y enmarcamiento.}

Diversos fenómenos psicológicos tienen influencia en la toma de decisiones económicas. Esos fenomenitos habitualmente se ubican en sesgos (biases) la heurística (heuristics) y el enmarcamiento (framing). A los efectos de este trabajo se entiende por sesgo a una predisposición hacia el error; por heurística las reglas de fuerte base empírica para tomar decisiones, y por enmarcamiento, la aproximación del fenómeno.

Los primeros estudios de Tverky y Kahneman revolucionaron el ámbito de la investigación sobre el juicio humano. El juicio bajo incertidumbre versa sobre una serie de heuristicos facilitadores y no en un procesamiento de tipo algorítmico. Si bien estos estudios introdujeron términos como heurística que refieren a principios sobre los que las personas se apoyan para reducir las complejas tareas de evaluar probabilidades y predecir valores para así formar juicios mas simples (Tversky \& Kahneman, 1973) no es sino hasta los estudios de Kahneman y Frederic (2002) en donde se extiende la palabra "heurística", como proceso cognitivo que va mas allá de juicio en condiciones de incertidumbre.

Los bien conocidos heuristicos que proponen son el de representatividad, el de disponibilidad y el de anclaje y ajuste. Los sesgos cognitivos según estos autores se encuentran agrupados y se corresponden a cada uno de los heuristicos

Su propuesta se instala en el campo de la psicología cognitiva como una alternativa por explicar qué procesos intervienen en este tipo de errores humanos.

Dentro de los sesgos y anomalías presentes en la literatura científica identificamos en este trabajo, en un sentido indicativo, algunos de los mismos.

El sesgo de sobreconfianza (overconfidence) se apoya en el entendido de que los seres humanos tendemos a sobrevalorar nuestras habilidades, nuestros conocimientos y nuestras perspectivas de futuro.(Camerer y Lovallo, 1999) Este sesgo en el pensamiento contribuye a que tendemos a creernos mejores pronosticadores de lo que realmente demuestra la evidencia empírica. Las personas sobreconfiadas si bien pueden ser muy listas, nunca son tanto como lo creen ser. Incluso un pasado de eventos exitosos de la misma índole puede contribuir a creencias de este tipo.

Camerer y Lovallo (1999) estudiaron que la sobreconfianza es uno de los factores intrínsecos del ser humano que se encuentra implicado en los elevados fracasos a la hora de emprender nuevos negocios. La sobreconfianza ha sido estudiada por sus influyentes implicancias en las decisiones económicas. Según Camerer (1997), uno de los aspectos que hace que contribuyen a ello, radica en la persistencia con que se mantiene este sesgo en el tiempo sin descender en intensidad. De estos estudios resulta que los inversores tienden persistentemente a sobreestimar los rendimientos de sus inversiones y subestimar los posibles resultados generados por la incertidumbre. Caliendo y Huang, (2007) demostraron esto para 3000 nuevos empresas en el cual $81 \%$ de la muestra contestó que su negocio tenia el $70 \%$ de probabilidad de éxito, mientras que el $33 \%$ reportó que ellos por seguro crecerán. Cinco años después de estas respuestas el $75 \%$ de los encuestados no sobrevivieron en sus negocios.

Con referencia a la sobreconfianza Fischhoff, (1988) sostiene que las causas no están claras como la evidencia que las demuestra. Sin embargo sus estudios señalan la preferencia a no pensar en resultados negativos.

La difícil predictibilidad que presenta en la actualidad la evolución de los mercados en interacción con la complejidad de los procesos cognitivos hace que el proceso decisorio este plagado de dificultades.

Otros sesgo a señalar el excesivo optimismo. En Psicología el excesivo optimismo refie-

\footnotetext{
${ }^{12}$ Arrow, por su parte habría recibido el premio Nobel en Economía, junto a Sir John Hicks en 1972 "por sus pioneras contribuciones a la teoría del equilibrio general en Economía y la teoría del bienestar"

${ }^{13}$ Allais recibiría en 1988 el premio Nobel en Economía "por sus pioneras contribuciones a la teoría de los mercados y de la eficiente utilización de los recursos".
} 
re a un procesamiento cognitivo que opera como una elevada tendencia a sobreestimar los resultados positivos o favorables y al mismo tiempo a subestimar los resultados desfavorables o considerarlos poco frecuentes. Se llega así a conclusiones que no se desprenden de la experiencia previa ni en cálculos estadísticos, sino que encierran creencias basadas en expectativas y en deseos del decicidor. Es la desabilidad por la obtención de resultados positivos lo que gatillan pensamientos englobados en el whishful thinking.

Este tipo de creencias se sustentan en una elevada expectativa de resultado y se focalizan en ello, por lo que no debemos entender que lo que se sobrevalora es la performance debido una autoefcicaia percibida. El resultado, si bien deriva de un cierto curso de acción, lo que es sobrevaluado es el pronóstico del primero, de ahí que el excesivo optimismo devenga un sesgo ya que realiza una zanja entre las acciones y los resultados de las mismas como si no actuaran de forma interdependientes. Lo que aquí también se juega es el sesgo de la ilusión de control, es decir, que el incremento del control percibido puede llevar un excesivo optimismo.

Tampoco debemos desatender que los pronósticos exagerados de consecuencias exitosas pueden provenir de sobrestimaciones internas de la persona, lo que estaría en línea con el citado de sobreconfianza.

Este sesgo es sumamente frecuente encontrarlo en los ámbitos de negocios y empresas, en el análisis de proyectos en los sectores tanto públicos como privados, en donde los administradores tienen una visión exagerada sobre el éxito de los resultados.

Abundan los estudios que han dado una evidencia más que suficiente del excesivo optimismo en diversas situaciones de índole empresarial. Una de ellas se ha detenido en el estudio del excesivo optimismo a la hora de hacer pronóstico sobre la venta de nuevos productos que se lanzan al mercado. (Tull, 1967)

Otro error que no será ajeno, es el sesgo de confirmación. Este describe la propensión a quedarnos prendidos de la información que confirme nuestras hipótesis, y a desestimar aquellos datos que pueden ponerlas en duda. Por ejemplo, las personas que ocupan cargos de alta administración exhiben frecuentemente este tipo de conductas en las cuales se detienen mucho tiempo buscando información que confirme sus puntos de vista y ponen a disposición todos aquellos recursos tal como les sea posible que contribuyan a esa meta. Cabe aclarar que los recursos son tanto cognitivos como materiales.

El sesgo de la maldición del conocimiento se le denomina a la tendencia a asumir que el conocimiento que tenemos sobre algo, también lo tienen los demás. Suponer que se maneja la misma información sobre un determinado tema puede llevar a conclusiones equivocadas. (Thaler, 2000)

El sesgo conocido como El efecto dotación se asocia con las creencias que las personas tienen a la hora de vender algo, demandando más dinero de lo que ellos están dispuestos a pagar para adquirirlo, Este fenómeno del efecto dotación y otro relacionado con este conocido como el sesgo del statu quo fue estudiado por Kahneman, Knetsch y Thaler (1991) en el trabajo Anomalies: endowment effect, loss aversion, and statu quo distortion. El efecto dotación es frecuentemente ejemplificado con el caso de un señor que ha comprado una caja de vinos de lata calidad que conserva en su bodega. En el entretiempo el valor de cada botella sube notablemente, habiendo el pagado menos de 20 dólares y ahora pasando a valer más de 200 cada una. Puede suceder que el señor ocasionalmente destape una de esas botellas en ocasión de estar con buenos amigos. Pero no vendería nunca su vino al precio del mercado corriente en el que esta ahora ni compraría una botella al precio que se encuentra ahora. En este caso, se esta frente al sesgo del efecto dotación. En cierto modo este efecto muestra un conservadurismo de la elección económica. En este caso una tendencia a confirmar una cierta elección de inversión antes que comprometerse en una nueva decisión. Es decir, si una persona tiende a atribuir un valor más alto a cuanto posee y a su statu quo entonces la decisión de cambiar se transforma en una posibilidad menos frecuente y más difícil.

Thaler (1992) describe este concepto de economía cognitiva denominado contabilidad mental (mental accounting) El siguiente ejemplo lo describe:

En Las Vegas durante su luna de miel una pareja se ha gastado su dinero que disponía para jugar en el casino. Al novio, una noche mientras estaba en la dormitorio se le cruza el numero 17 por la mente de una forma absolutamente inevitable. Busca más dinero y encuentra \$5. Baja al casino y comienza a jugar a la 
ruleta. En la primera ronda entra el 17 y el 35 , y el novio gana $\$ 175$, continua jugando y gana $\$ 6125$. El numero 17 vuelve a quedarse en la mesa y el afortunado novio gana $\$ 7.5$ millones. El gerente del casino le comunica que si vuelve a entrar el 17, el casino no cuenta con más dinero como para pagarle, por lo que el novio se va enojado a otro casino. Allí comienza a jugar y sale nuevamente el numero 17 ganando esta vez \$262 millones. Extasiado deja sus millones correr hasta que finalmente pierde todo la cantidad cuando sale el numero 18. Defraudado y deprimido vuelve al hotel.

Cuando entra en la habitación su esposa le pregunta "Donde estabas?"'

-"Jugando a la ruleta"

-"como te fue?" le vuelve a preguntar ella

- "Nada mal, perdí 5 dólares"

Esta famosa historia de Nevada intenta contestar la pregunta de porque los casinos siempre ganan. Uno de las razones que es la estructura de juegos está sesgada probabilisticámente a favor de la banca cuando opera la ley de los grandes números, La otra razón que es que quienes apuestan piensan como el "afortunado" novio, que la pérdida solo se limita a $\$ 5$. Esta creencia supone no poder visualizar el dinero como una cantidad real lo que se asocia con otra creencia: de que las pérdidas tampoco son reales. El novio ve su ganancia como algo totalmente diferente y por tanto se siente animado a continuar realizando extravagantes apuestas. Esta es la tendencia a realizar cuentas mentales separadas, categorizando y tratando el dinero de forma diferente dependiendo de donde viene y como lo obtengo, para que lo guardo, y en que lo gasto, es decir, dependiendo en que cuenta mental se encuentre. Este valor diferente que se le cada a cada una se produce porque se le da un significado diferente a cada dólar. Valorar algunos dólares como si fueran distintos de otros es uno de los errores económicos mas frecuentes en la vida cotidiana (Thaler, 1992)

Otra anomalía es la denominada maldición del ganador (Winner's curse) la cual se describe a partir del siguiente ejemplo por Thaler (1992) en el texto del mismo nombre:

Supongamos que los derechos de perforación de petróleo en una parcela de tierra varias empresas se encuentran interesadas. Cada firma oferente hace unas estimaciones al valor de los derechos en base a estimaciones de los expertos. Se pregunta Thaler "¿qué sucederá en la licitación?". Existen estimaciones por parte de los oferentes pero todas ellas generadas con serias dificultades por lo que algunas pueden ser demasiado altas y otras demasiado bajas. La compañía que compre los derechos puede ser una de las que hizo de las estimaciones más altas de cantidad de petróleo. En ese caso, el ganador en realidad será un perdedor. Puede ser considerado ha sido "maldito" en dos sentidos siguiendo el ejemplo por Thaler. En primer término, que el ganador excedió el valor de la oferta y la empresa perdió riqueza, o en segundo término el valor de la reservas petrolíferas es menor a las estimaciones realizadas por los expertos con lo cual la firma ganadora estará desconforme. Esta situación no se daría si los oferentes fueran racionales y se tuviera la total información por lo cual va a constituir una anomalía.

Estos sesgos no actúan aisladamente sino que muchas de las veces no resulta tarea sencilla desenmarañar cual es el que se encuentra operando. Justamente lo que los caracteriza y por lo que han sido investigados desde distintas disciplinas es por su carácter persistente y sistemático. La tarea de mitigar los sesgos no resulta tarea sencilla ya que el conocimiento de los procesos que se ponen en juego no garantiza el cambio (debiasing)

Por ultimo, mencionamos el efecto enmarcamiento (framing effect) que es a través del cual se efectúa una descripción del tema a decidir. Este efecto enmarcamiento afecta a quienes toman decisiones al establecer una impostación del marco en el que la decisión queda inserta.

Tversky y Kahneman en su Prospect Theory que exponemos más adelante otorgan al efecto enmarcamiento un papel importante en sus desarrollos de la función de valor.

Dos fenómenos de este efecto se destacan en la citada teoría de los mencionados autores. Ellos son: aversion al riesgo y aversión a una pérdida segura.

La aversión al resigo dice relación con el hecho que, psicológicamente los agentes económicos le otorgan mas importancia a una perdida que a una ganancia de la misma magnitud.

Supónganse que una persona tiene iguales probabilidades, esto es $50 \%$ en cada caso de perder $\$ 60$ o ganar una determinada cifra. La pregunta es ¿cuánto tiene que ser el monto de la probable ganancia? \$150, \$250, \$500 ó que cifra? O sea hasta qué monto puede descender 
esa potencial ganancia de forma tal de obtener una indiferencia de aceptar o rechazar la oportunidad de correr el riesgo. Numerosos estudios experimentales ubican a la ganancia que obtuvo mas respuesta en torno a $\$ 150$. Ello significa que las personas valorar la perdida de $\$ 60$ dos veces y media mas que una ganancia probable $(\$ 150 / 60=2.5)$

El segundo fenómeno de efecto enmarcamiento por Tversky y Kaheman es el de aversion a una perdida segura. El mismo en este caso dice relación que los sujetos económicos aceptaran un curso de acción riesgoso en un intento por evitar una perdida segura.

Supongamos que un sujeto se ve enfrentado a optar por una perdida segura de $\$ 70$ y una opción riesgosa. La alternativa riesgosa es una situación con el $70 \%$ de probabilidad de ganar $\$ 100$ y el $30 \%$ de ganar 0 . Cuál será la opción que tomara? Esto es, la perdida segura o la opción riesgosa? La mayor parte los experimentos muestras a los sujetos inclinándose por la opción riesgosa.

\section{INTRODUCIENDO LA PROSPECT THEORY DE TVERSKY Y KAHNEMAN}

Esta introducción a la Prospect Theory de Tversky y Kahneman se efectuará en dos aproximaciones. La primera de ellas tendrá un carácter más intuitivo, con miras a facilitar el tránsito en la segunda aproximación, de mayor densidad formal.

\subsection{Aproximación intuitiva}

Se han repasado en este trabajo varias anomalías en la vida económica cotidiana, que tienen en común algunos aspectos característicos que son coincidentes en ellos. Se trata de ser el reflejo de alguna peculiaridad cognitiva que contradicen las decisiones "reales" del hombre común, que vive en un mundo cambiante, cuando no caótico y hasta en contraste con las decisiones habitualmente atribuidas al Homo Economicus el que transcurre en una abstracción, con precisión propia de modelos económicos.

Daniel Kahneman y Amos Tversky desarrollaron una teoría descriptiva de la toma de decisiones de los seres humanos. En su teoría resaltan expresamente que el hombre "común", tiene un modelo perceptivo estructura- do de forma de conocer los cambios y las diferencias, más que valorar las dimensiones absolutas.

En una obra de arte, un valor puede parecer mas luminoso según se coloque al lado de uno u otro color diferente.

El cuadro que sigue lo pone de relieve:

\section{Figura 1}

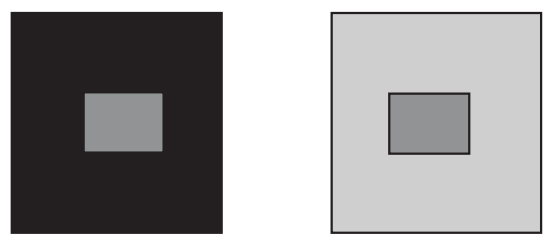

Fuente: Kahneman (2002)

Se advierte que el cuadrado pequeño si bien tiene el mismo color, aparece con luminosidad diferente según en que cuadrado mayor esté inserto. La búsqueda de una dependencia en una referencia en la toma de decisiones es lo que lleva a ambos autores a realizar esta analogía con los procesos perceptivos visuales.

Las diferencias de luminosidad son dimensiones relativas y no absolutas.

Estos aspectos intuitivos que se vienen desarrollando, Kahneman y Tversky lo aplican a cualidades no sensoriales como la riqueza o el prestigio o la salud.

Sobre este punto Kahneman (2002) señala: "El valor está dado por la diferencia entre los estados económicos y no están dados por los datos presentado en si mismos".

Los estudios experimentales de Tversky y Kahneman los hace concluir los individuos son aversos al riesgo, cuando se trata de situaciones en el ámbito de las ganancias y por su parte, son buscadores de riesgos cuando están en el ámbito de las pérdidas.

Se advierte en la clásica grafica que expusieron ambos autores.

De ella se muestra que ganar 200 unidades monetarias produce un placer, una utilidad de 25 unidades. Si se observa el cuadrante inferior izquierdo se advierte que perder 200 unidades monetarias produce una utilidad negativa de de mas de 50.

De esta forma la mayor parte de nosotros probablemente aceptemos la apuesta cuando se puede ganar 200/250 o, también perder 100 . 
Figura 2

Función de valor según Tversky y Kahneman

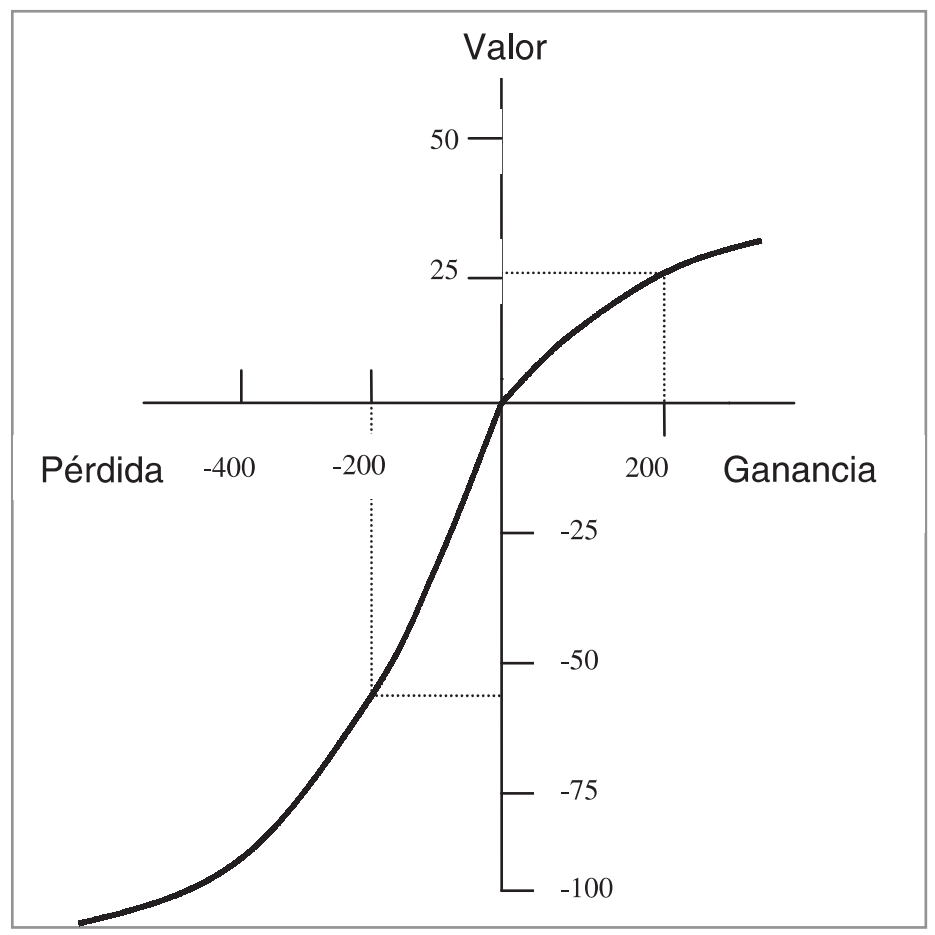

Fuente: Kahneman (2002)

\subsection{Aproximación formal}

La teoría de la utilidad esperada (UE) (Bernoulli, 1738, Von Neumann y Morgenstern, 1947) dominó por un largo período de tiempo el estudio de la teoría de las decisiones en condiciones de riesgo.

Tversky y Kahneman (1987) profundizarán y darán nuevas dimensiones a las ideas de racionalidad acotada iniciada por Simon y también a las violaciones a la utilidad esperada expuestas por Allais.

En "Rational Choice and the Framing of Decisions" (1986) analizan el proceso de selección en condiciones inciertas como distinguiendo dos aspectos; en primer lugar, la estructuración de los eventos tendrá la representación mental de ellos y, en segundo lugar, una fase de valuación.

En este proceso de representación mental, se resalta el marco (framing) en el cual el individuo coloca la elección y que, a igualdad de todas las condiciones determina un comportamiento diferente.

La representación mental de los eventos se transforma en un punto crucial de la decisión. Demuestran en numerosos experimen- tos cómo un individuo muestra una actitud diferente ante el riesgo según el contexto en que se sitúa.

El siguiente es el clásico ejemplo de Tversky y Kahneman sobre el comportamiento de numerosos sujetos, ante las siguientes propuestas de elección:

Problema 1

- Supongamos de ser más rico de 300 dólares respecto de lo que se es hoy. Se debe elegir entre:

- A: una ganancia segura de 100 dólares

- B: $50 \%$ de probabilidad de obtener 200 dólares y $50 \%$ de no obtener nada.

\section{Problema 2}

- Supongamos de ser más rico de 500 dólares respecto a hoy. Se debe elegir entre:

- C: una pérdida segura de 100 dólares.

- D: $50 \%$ de probabilidad de no perder nada y $50 \%$ de perder 200 dólares.

La mayoría se inclinaron en el problema uno por la elección A que representa una ganancia segura (elección A), mientras que efectuada la elección favorable al riesgo se inclina por la elección $\mathrm{D}$ del problema 2. 
Se observa que la mayoría, que efectúan las elecciones $A$ y $D$, violan la teoría de la utilidad esperada, en particular, el axioma de la independencia.

Ambos problemas, en términos de la utilidad esperada, son el mismo problema. La riqueza a disposición del sujeto, después de que la elección haya sido efectuada es:

\section{Problema 1}

- Caso A: 400 con prob. $=1$

- Caso B: 300 con probab. $=0.5$,

o 500 con probab. $=0.5$.

Problema 2

- Caso C: 400 con prob. $=1$

- Caso D: 300 con prob. $=0.5$,

o con 550 prob. $=0.5$

En condiciones de incertidumbre se tiende a evitar mayormente el riesgo cuando se está en condiciones de ganancia. Sin embargo, cuando se está en condiciones de pérdida, son buscadores de riesgo. Estas situaciones se observan en problemas que en términos de la utilidad esperada es el mismo problema.

Este framing effect no solo influye sobre el tema de la utilidad esperada sino según Tversky y Kahneman, (1979)

"sino sobre prácticamente todos los modelos de elección basados sobre otras teorías normativas".

Los estudios de Tversky y Kahneman muestran en los individuos anomalías cognitivas, que los separan fuertemente de la racionalidad implícita en la teoría de la utilidad esperada. Se separan en sus decisiones de lo que haría el "hombre de Chicago". Según los autores, quienes toman las decisiones tienen problemas en la obtención y utilización de la información, en la formación de percepciones coherentes y por otra parte utilizan heurísticas para sus decisiones que pueden ser incompatibles con las hipótesis de maximizar las preferencias y son individuos sensibles.

En estas aproximaciones de fuerte impronta cognitiva, ponen un especial énfasis en la comprensión de los elementos de la decisión, en la forma que se ubican las situaciones así como la determinación de los valores. En economía, habitualmente, se pone énfasis en el camino que va desde los inputs de información a la elección.
Así, las preferencias se ubican como previas al análisis $\mathrm{y}$, el proceso como una "caja negra". Según los modelos económicos neoclásicos, el consumidor se comporta "como si" existiera una racionalidad, tanto de la percepción, como de las preferencias y del proceso.

En su versión original, (Original Prospect Theory, OPT) fue expuesta por Kahneman y Tversky en 1979. Años después, en 1992 Tversky y Kahneman efectúan ampliaciones a sus proposiciones originales incorporando rangos y signos en la utilidad. Esta nueva aproximación de 1992 se conoce como la Cumulative Prospect Theory (CPT) que permitió desarrollar las paradojas previamente expuestas por Allais (1953).

La Prospect Theory pasaría a ser el centro de los estudios empíricos sobre la toma de decisiones.

En su Cumulative Prospect Theory, Tversky y Kahneman efectúan un tratamiento separado de las ganancias y de las pérdidas. Por otra parte postulan la existencia de dos funciones; la función de valor y la función de ponderación (esto es del peso de las decisiones)

En su desarrollo formal básico se supone que un grupo/juego está compuesto por $m+n+1$ resultados monetarios tales que $x_{-m}<\ldots<x_{0}<\ldots<x_{n}$ que ocurren con posibilidades dadas $p_{-m}, \ldots p_{n}$ respectivamente. Los juegos pueden ser denotados por los pares $(x ; p)$ en donde $x=\left(x_{-m}, \ldots, x_{n}\right)$ y $p=\left(p_{-m}, \ldots, p_{n}\right)$, En la clásica Teoría de la Utilidad la utilidad esperada de este juego vendría dada por :

$$
E U(x ; p)=\sum p_{i} \mu\left(x_{i}\right)
$$

La CPT, hace una aproximación diferente definiendo

$$
\begin{aligned}
& V^{+}(x ; p)=g\left(p_{n}\right) u\left(x_{n}\right)+\sum_{k=1}^{n}\left[g\left(\sum_{j=0}^{k} p_{n-j}\right)-g\left(\sum_{j=0}^{k-1} p_{n-j}\right)\right] u\left(x_{n-k}\right), \\
& V^{-}(x ; p)=g\left(p_{n}\right) u\left(x_{-m}\right)+\sum_{k=1}^{m}\left[g\left(\sum_{j=0}^{k} p_{-(m-j)}\right)-g\left(\sum_{j=0}^{k-1} p_{-(m-j)}\right)\right] u\left(x_{-(m-k)}\right) . \\
& \text { El valor de la preferencia del juego }(\boldsymbol{x} ; \boldsymbol{p}) \\
& \text { está dado por: }
\end{aligned}
$$

$$
V(x ; p)=V^{+}(x ; p)+V^{-}(x ; p)
$$


La Expresión $V^{+}$mide la contribución de las ganancias y $V^{-}$mide la contribución de las pérdidas.

La Función $g(p)$ es la función de probabilidad ponderada que se supone crece de $g(0)=0$ y $g(1)=1$ y $\mu(x)$ es la función de utilidad (o valor) que se supone que se incrementa a partir de $\mu(0)=0$

La Función de utilidad propuesta por Tversky y Kahneman es:

$$
u(x)=\left\{\begin{array}{cc}
x^{\alpha} & x \geq 0 \\
\text { para } & \\
-\lambda(-x)^{\alpha} & x<0
\end{array}\right.
$$

Para $\alpha$ la función de utilidad mostrará aversión al riesgo sobre las ganancias y para las pérdidas búsqueda de riesgo. Por otra parte si....., el coeficiente de aversión al riesgo, es mayor que 1 , los individuos son más sensibles a las pérdidas que a las ganancias.

La función de utilidad ponderada usada por Tversky y Kahneman (1992) fue:

$$
g(p)=\frac{p^{\gamma}}{\left[p^{\gamma}+(1-p)^{\gamma}\right]^{\frac{1}{\gamma}}}
$$

que gráficamente es:

\section{Figura 3}

Función de utilidad ponderada de Tversky y Kahneman

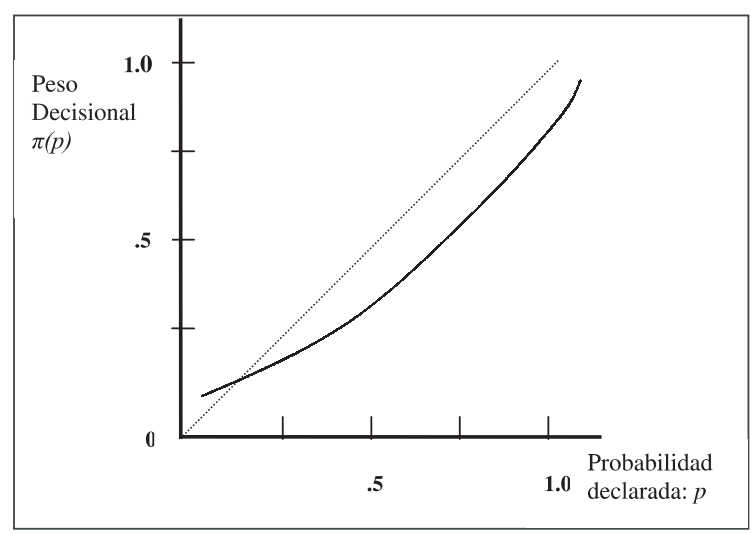

Fuente: Kahneman (2002)
Se advierte que a diferencia que la EU tenía con la función de ponderaciones de la Cumulative Prospect Theory da más peso a la los extremos de resultados cuando sus probabilidades son bajas y da menos peso cuando sus probabilidades son bajas y da menos peso cuando sus probabilidades son altas.

Cuando las distribuciones de probabilidad tienen solo dos resultados, el resultado extremo es sub-ponderado, por defecto, el extremo menor de resultados es sobreponderado, lo que es consistente con la ponderación sobre aversión al riesgo sobre ganancias y búsqueda de riesgo sobre pérdidas.

La Figura muestra que decrecientes en $\gamma$, causan que la función ponderada se vuelva más nueva y que crece la recta de $45^{\circ}$.

Se han efectuado diversos estudios que suministran parámetros para las funciones diferentes.

Las estimaciones de Tversky y Kahneman (1992) fueron

$\alpha=0,88 ; \lambda=2,25$ y $\gamma=0,61$ para ganancias y $\gamma=0,69$ para pérdidas. Camerer y Ho (1994) concluyeron en $\alpha=0,32$ y $\gamma=0,56 \mathrm{Wu}$ y González (1996) llegara a á $=0,52$ y $\gamma=0,74$.

Importantes trabajos han contribuido al desarrollo teórico y empírico de la Prospect Theory (Abdellaoui, 2000; 2002; Camerer, 1989; 1992; 1998; Decidue y Wakker, 2001; González y Wu, 1999; Karni y Safra; 1987; Luce, 2000; 2001; Luce y Fishburn, 1991, 1995; Luce y Narens, 1985; Machina, 1982; Prelec, 1998; Quiggin, 1982; 1985; 1993; Schmeidler, 1989; Starmer y Sugden, 1989; Tversky y Wakker, 1995; Yaari, 1987; von Winterfeldt, 1997; Wakker, 1994; 1996; 2001; Wakker, Erev, y Weber, 1994; Wu y González, 1996; 1998; 1999). Debido a estos éxitos, la Cumulative Prospect Theory ha sido recomendada como el nuevo estándar para el análisis económico (Camerer, 1998; Starmer, 2000).

La Cumulative Prospect Theory ha sufrido, sin embargo, por esta variación de parámetros determinadas críticas por sus efectos sobre los premios por el riesgo diferentes que resultan.

Sin embargo, la evidencia ha estado acumulando en estos últimos años que viola sistemáticamente ambas versiones de la teoría de la perspectiva. Algunos autores han criticado CPT (Baltussen, Post, \&y Vilet, 2004; Barron \& Erev, 2003; Brandstaetter, Gigerenzer \& Hertwig, 2006; González \& Wu, 2003; González 
-Vallejo, 2002; Hertwig, Barron, Weber, \& Erev, 2004; Humphrey, 1995; Marley \& Luce, 2005; Neilson \& Stowe, 2002; Levy \& Levy, 2002; Lopes \& Oden, 1999; Luce, 2000; Payne, 2005; Starmer \& Sugden, 1993; Starmer, 1999, 2000; Weber \& Kirsner, 1997; Wu, 1994; Wu \& González 1999; Wu \& Markle, 2005; Wu, Zhang, \& Abdelloui, 2005).

No todas las críticas de la Cummulative Prospect Theory se han recibido sin controversia (Baucells y Heukamp, 2004; Fox \& Hadar, 2006; Rieger y Wang, (en prensa); Wakker, 2003), sin embargo, algunos concluyen que Cumulative Prospect Theory es la "mejor", aunque imperfecta descripción de la toma de decisiones bajo riesgo e incertidumbre (Camerer, 1998; Starmer, 2000; Harless y Camerer, 1994; Wu, Zhang y González, 2004).

\subsection{Cumulative Prospect Theory y Teo- ría de la Utilidad Esperada}

La Cumulative Prospect Theory y la teoría de la utilidad esperada presentan características diferenciales. Tres de ellas son de especial significación.

En primer término, en la Cumulative Prospect Theory el decididor no está interesado en el valor final, sino en el cambio de estado con respecto a un cierto nivel de referencia, que está determinado por un proceso cognitivo.

En segundo lugar, la función de valor tiene, como se ha visto, una forma de "S" como se ha visto, siendo distintas la actitud ante el riesgo de los individuos frente a las ganancias que frente a las pérdidas.

Por último, en la teoría de la utilidad esperada, la utilidad de cada éxito esperado es ponderado por su probabilidad. En la Cumulative Prospect Theory, el valor de cada cambio de la utilidad es multiplicado por la función de ponderación o el peso de la decisión.

El peso de la decisión no es la probabilidad sino transformaciones de la probabilidad.

Como señalan los autores "miden el impacto de los eventos sobre la deseabilidad de la prospectiva y no simplemente la probabilidad percibida de los eventos" (Kahneman, 2002). En el impacto de los eventos sobre la deseabilidad prospectiva, aparecen sesgos, el uso de la heurística y el enmarcamiento.

La Cumulative Prospect Theory propone una teoría para describir la toma de decisiones reales y no para definir una elección racional y, por tanto, busca incorporar violaciones a la racionalidad perfecta cuando ellas tienen lugar.

Los significativos aportes de Tversky y Kahneman a través de su proficua labor investigativa que en la Prospect Theory resumen una de sus más notables ejemplos, no deben poco a Simon y a Allais. El primero, al señalar con singular lucidez que los economistas no pueden quedarse satisfechos de la capacidad predictiva de la teoría de la utilidad esperada. Y, en cuanto a Allais, la pérdida de importancia de las probabilidades, a la hora de estructurar la función de valor.

Kahneman y Tversky no tienen en sus objetivos mostrar lo inadecuada que es la teoría de la utilidad esperada como teoría normativa, sino en mostrar que es inadecuada empíricamente y por tanto predictiva. He aquí la pars destruens del proyecto cognitivo aplicado a la economía. Mientras que su pars construens, consiste en integrar a los modelos neoclásicos, los resultados de la evidencia experimental anómala y de los principios psicológicos cognitivos descubiertos por vía de experimentos que contribuyen a explicarla.

Buscando sintetizar, lejos de haber "demostrado la irracionalidad humana" como han sugerido estudios superficiales, Tversky y Kahneman han tomado distancia de la noción irrealística de racionalidad perfecta indicando las vías para construir modelos en base a racionalidad acotada.

\section{UN ASOMO A UN NUEVO PASO: LA NEUROECONOMÍA}

En la economía neoclásica, los detalles de cómo funciona el cerebro humano en la toma de decisiones no fueron tomados en consideración.

Los aportes cognitivos son sustanciales en el esclarecimiento de la toma de decisiones y así se expuso el pensamiento de Simon, Allais Kahenman y Tversky.

Si bien el objetivo de este trabajo es repasar el aporte cognitivo en la toma de decisiones es útil mencionar aunque sea someramente nuevos pasos que la ciencia viene dando en el proceso de mejor explicar la toma de decisiones.

Los estudios actuales acerca de la especialización cerebral y su funcionamiento por regiones realizan aportes en relación de la medición de las emociones y los pensamientos (Camerer, Lowenstein y Prelec, 2005). La aparición de las emociones en el terreno de la toma de decisio- 
nes es conocido como el heurístico de afecto, en el cual las decisiones están impulsadas por emociones. Esto lleva a que las decisiones finales que involucran riesgos e incertidumbres resulten de la resolución entre los aspectos emocionales y cognitivos en el individuo.

El estudio del actividad cerebral y de las emociones van abriendo nuevos y promisorios campos para la economía no solo en cuanto a riesgo e incertidumbre sino también en el análisis del mercado de trabajo, elecciones interpersonales, por mencionar algunos (frederick, Lowenstein y O’Domoghue, 2002; McConnell y Leibold, 2001)

\section{CONSIDERACIONES FINALES}

A la hora de producir conocimiento aparece la multidisciplinariedad, esto es, cuando el objeto de estudio es tratado desde distintas disciplinas al mismo tiempo. Seguramente la multidisciplinariedad siempre agrega algo nuevo, pero rara vez es idónea para producir avances trascendentes en el conocimiento.

La interdisciplinariedad, por el contrario, intenta dar respuesta a esa demanda no satisfecha de crear nuevos conocimientos. En este caso se produce una transferencia de métodos y hallazgos entre distintas disciplinas para alcanzar nueva proposiciones que expliquen más adecuadamente el objeto de estudio.

Este trabajo busca introducir al lector en un ejemplo paradigmático de interdisciplinariedad; en este caso entre psicología cognitiva y toma de decisiones económicas. Los hallazgos interdisciplinarios de Simon, Allais y de Tversky y Kahneman entre ambas disciplinas ocupan hoy día un lugar de singular prestigio en el seno de la comunidad académica internacional. En particular Tversky y Kahneman, dos psicólogos cognitivos han contribuido sustancialmente a explicar el proceso de decisiones económicas, incorporando hallazgos provenientes de la psicología cognitiva. Contribuyeron de esta forma a tener mejores predicciones, así como disminuir el debiasing decisorio.

El esfuerzo académica en esta línea interdisciplinaria entre psicología cognitiva y economía continúa a un ritmo acelerado y también profundo. A ello se le debe agregar los nuevos estudios del cerebro que arrojan luz sobre las emociones gestando una nueva ciencia: la neuroeconomía. De este esfuerzo seguramente se verán nuevos frutos en la interminable tarea de la explicación científica de la realidad.

\section{REFERENCIAS}

Abdellaoui, M. (2000). Parameter-free elicitation of utility and probability weighting functions. Management Science, 46, 1497-1512.

Abdellaoui, M. (2002). A genuine rank-dependent generalization of the von NeumannMorgenstern expected utility theorem. Econometrica, 70, 717-736

Allais, M. (1953). Le comportement de I'homme rationnel devant le risque; Critique des postulats et axiomes de l'École Américaine Econometrica 21, pp.503-54

Arrow, K.J. (1978). The Future and the Present in Economic Life, Economic Enquiry, pp. 157-170.

Arrow, K. J. (1965). Aspects of the Theory of Risk Bearing. Helsinski. Yrjo Hahnsson Foundation.

Baltussen, G., Post, T., Van Vliet, P. (2004). Violations of CPT in mixed gambles. Working Paper, July, 2004., Available from Pim van Vliet, Erasmus University Rotterdam, P.O. Box 1738, 3000 DR Rotterdam, The Netherlands.

Barron, G., Erev, I. (2003). Small feedback based decisions and their limited correspondence to description based decisions. Journal of Behavioral Decision Making, 16, 215-233.

Baucells, M., Heukamp, F. H. (2004). Stochastic dominance and cumulative prospect theory. Working paper, dated June, 2004., Available from Manel Baucells, IESE Business School, University of Navarra, Barcelona, SPAIN.

Bernoulli D. (1738). Specimen theoriae novae de mensurea sortis, Commentarii Academiae Scientiarum Imperialis Patropolitanae, vol. 5, pp. 175-192 (trad. ingl di L. Sommer, "Exposition of a New Theory on the Measurement of Risk", Econometrica, 1954, vol. 22, pp. 23-36).

Brandstätter E, Gigerenzer G., Hertwig R, (2006). The priority heuristic: Choices without tradeoffs. Psychological Review, 113, 409-432.

Caliendo, Frank and Huang, Kevin X.D., (2007) Overconfidence in Financial Markets and Consumption Over the Life Cycle FRB of Philadelphia Working Paper No. 07-3

Camerer, C. F. (1989). An experimental test of several generalized utility theories. Journal of Risk and Uncertainty, 2, 61-104.

Camerer, C. F. (1992). Recent tests of generalizations of expected utility theory. In W. Edwards (Eds.), Utility theories: Measurements and applications (pp. 207251). Boston: Kluwer Academic Publishers

Camerer C, Lovallo D, (1999)Overconfidence and Excess Entry: An Experimental Approach The American Economic Review, Vol. 89, No. 1 pp. 306-318

Camerer C, Lowenstein G, Rabin M (2003) Advances in behavioral Economics. Princeton University Press

Camerer, C (1998). Bounded rationality in individual decision making. Experimental Economics, 1, 163-183. 
Camerer, C Lowenstein, G y Prelec, O (2005) "Neuroenconmics: how neuroscience can inform Economics" Journal of Economic Literature.

Diecidue E., Wakker P. P. (2001). On the intuition of rank-dependent utility. Journal of Risk and Uncertainty, 23, 281-298.

Fernández Álvarez, H (1992) Fundamentos de Modelo Integrativo en psicoterpia Paidos,

Gardner, H. (1988) La nueva ciencia de la mente, Paidos

Gigerenzer G, Selten E. Eds, (2001) Bounded rationality: The adaptative toolbox. MIT Press, Cambridge, MA London.

Fox C. R., Hadar, L. (2006). Decisions from experience $=$ sampling error + prospect theory: Reconsidering Hertwig, Barron, Weber \& Erev (2004). Judgment and Decision Making, 1, 159161.

Friedman M, (1953). Essays in Positive Economics, Chicago University Press, Chicago (trad. It. In Método, consumo e moneta, II Mulino, Bologna 1996, cap.1).

Friedman M, e Savage L.J, (1952). The Expected Utility Hypotesis and the Measurability of Utility, Journal of Political Economy, 60,6 (trad. it. In Metodo, consumo e moneta, II Mulino, Bologna 1996, cap.11).

Federick S, Lowenstein G, O'Domoghue T (2002) "Time discontinuing and time preference: a critical review" Journal of Economic Literature.

Gonzalez R, Wu G. (1999). On the shape of the probability weighting function. Cognitive Psychology, 38, 129-166.

Gonzalez R, Wu G. (2003). Composition rules in original and cumulative prospect theory. Working Manuscript dated 8-14-03.

González-Vallejo, C. (2002). Making trade-offs: A probabilistic and context-sensitive model of choice behavior. Psychological Review, 109, 137-155.

Harless, D. W., \& Camerer, C. F. (1994). The predictive utility of generalized expected utility theories. Econometrica, 62, 1251-1290.

Hertwig, R., Barron, G., Weber, E. U., \& Erev, I. (2004). Decisions from experience and the effect of rare events in risky choices. Psychological Science, 15, 534-539.

Humphrey, S. J. (1995). Regret aversion or eventsplitting effects? More evidence under risk and uncertainty. Journal of risk and uncertainty, 11, 263-274.

James, W (1890) Principios de Psicología México FCE 1989

Kahneman, D,. E Tversky A. (1973). On the Psychology of prediction. Psychological Review 80 237-251

Kahneman, D. Tversky, A (1986) Rational Choice and the Framing of Decisions Journal of Business

Kahneman, D,. E Tversky A. (1979). Prospect Theory: An Analysis of Decisions Under Risk, Econometrica, 47, pp. 313-327.
Kahneman D. Frederick, S (2002) Representativeness revisited: attribute subsitution on intutitive judgment, New York, Cambridge University Press

Kahneman, D Knetsch, L y Thaler, R. (1991) Anomalies: Endowment effect, loss aversion, and statu quo distortion. Journal of Economic Perspectives.

Kahneman, D (2002) Maps of Bounded Rationality: A perspective on intuitive judgment and choice. Prize Lecture. Nobel Foundation

Karni, E., Safra, Z. (1987). Preference reversal and the observability of preferences by experimental methods. Econometrica, 55, 675-685.

Kuhn, J. (1962). The Structure of Scientific Revolutions. Chicago: Chicago University Press.

Levy, M., Levy, H. (2002). Prospect Theory: Much ado about nothing. Management Science, 48, 1334-1349.

Lopes, L. L., Oden, G. C. (1999). The role of aspiration level in risky choice: A comparison of cumulative prospect theory and SP/A theory. Journal of Mathematical Psychology, 43, 286-313.

Luce, R. D. (2000). Utility of gains and losses: Measurement-theoretical and experimental approaches. Mahwah, NJ: Lawrence Erlbaum Associates.

Luce, R. D. (2001). Reduction invariance and Prelec's weighting functions. Journal of Mathematical Psychology, 45, 167-179.

Luce, R. D., Fishburn, P. C. (1991). Rank- and signdependent linear utility models for finite first order gambles. Journal of Risk and Uncertainty, 4, 29-59.

Luce, R. D., Fishburn, P. C. (1995). A note on deriving rank-dependent utility using additive joint receipts. Journal of Risk and Uncertainty, 11, 5-16.

Luce, R. D., Narens, L. (1985). Classification of concatenation measurement structures according to scale type. Journal of Mathematical Psychology, 29, 1-72.

Machina, M. J. (1982). Expected utility analysis without the independence axiom. Econometrica, 50, 277-323.

Marley, A. A. J., Luce, R. D. (2005). Independence properties vis-à-vis several utility representations. Theory and Decision, 58, 77-143.

McConnell, A. R., \& Leibold, J. M. (2001).Relations among the implicit association test discriminatory behavior and explicit measures radical attitudes. Journal of experimental Social psychology

Newell, A., Shaw, J. C. y Simon, H. A. (1958). Elements of a theory of human problem solving. Psychological Review, 65

Neilson, W., Stowe, J. (2002). A further examination of cumulative prospect theory parameterizations. Journal of Risk and Uncertainty, 24(1), 31-46.

Neisser, U (1999) Psicología cognoscitiva, México: Trillas 
Payne, J. W. (2005). It is whether you win or lose: The importance of the overall probabilities of winning or losing in risky choice. Journal of Risk and Uncertainty, 30, 5-19.

Pratt, J. W. (1964). "Risk Aversion in the Small and in the Large". Econometrica, Vol. 32, p. 22-36.

Prelec, D. (1998). The probability weighting function. Econometrica, 66, 497-527.

Quiggin, J. (1982). A theory of anticipated utility. Journal of Economic Behavior and Organization, 3, 324-345.

Quiggin, J. (1985). Subjective utility, anticipated utility, and the Allais paradox. Organizational Behavior and Human Decision Processes, 35, 94-101.

Quiggin, J. (1993). Generalized expected utility theory: The rank-dependent model. Boston: Kluwer.

Rieger, M. O., Wang, M. (in press). What is behind the priority heuristic: A mathematical analysis and comment on Brandstätter, Gigerenzer, and Hertwig (2006). Psychological Review, in press

Robbins L.C. (1932). An Essay on the Nature and significance of Economic Science, Macmillan, New York ( $2^{\text {nd }}$. Ed. Rivista ed estesa, 1935; trad it. Saggio sul la natura e il significato della scienza economica, Utet, Torino 1953).

Samuelson, Paul A. y Nordhaus W.(1985). Economics Mc Graw-Hill, New York.

Schwartz H, (1998) rationality gone awry? Decision making inconsistency with Economic and Financial Theory. Praeger westfort CT London.

Schmeidler, D. (1989). Subjective probability and expected utility without additivity. Econometrica, 57, 571-587.

Simon, H. A. (1947). Administrative Behavior, New York, Macmillan.

Simon, H.A. (1957). Models of Man, Wiley, New York.

Simon, H.A. (1969). The Sciences of the Artificial (Carl Taylor Compton Lectures), Mit Press, Cambridge (Ma.) (trad. it. Le scienze dell'artificialle, II Mulino, Bologna 1988).

Simon, H.A. (1971). Theories of Bounded Rationality, in McGuire B. E Radner R. (a cura di) Decision and Organization, North-Holland, Amsterdam.

Simon H.A. (1979). Rational Decision Making in Business Organization, American Economic Review, vol. LXIX, pp. 493-513.

Simon, H.A. (1991a). Models of My Life, Basic Books, New York (trad it. Modelli per la mia vita, Rizzoli, Milano 1992).

Simon, H.A. (1991b). Bounded Rationality and Organizational Learning, Organization Science, 2, pp. 125-134.

Simon H.A., Newell A. (1972). Human Problem Solving, Prentice-Hall, Englewood Cliffs (N.J.).

Starmer, C. (1999). Cycling with rules of thumb: An experimental test for a new form of non-transitive behavior. Theory and Decision, 46, 141-158.

Starmer, C. (2000). Developments in non-expected utility theory: The hunt for a descriptive theory of choice under risk. Journal of Economic Literature, 38, 332-382.

Starmer, C., Sugden, R. (1989). Violations of the independence axiom in common ratio problems: An experimental test of some competing hypotheses. Annals of Operations Research, 19, 79-101.

Sternberg, R (2005) Cognitive Psychology. Thomson

Thaler, R. (2000) From homo Economicus to homo Sapiens. Journal of Economic Perspective. Vol 14

Thaler, R (1992) The Winner's Curse. Princeton University Press

Tull, A (1967) The relationship of actual and predicted sales and profits in the new-product introductions. Journal of Business

Tversky A., Kahneman D. (1987). "Rational Choice and the Framing of Decisions, in Hogart R.M. e Reder M." Rational Choice -The Contrast between Economics and Psychology, The University of Chicago Press, Chicago (trad. it. In Egidi M. E Turvani M. (a cura di), Le ragioni delle organizzazioni economiche, Rosenberg \& Sellier, Torino 1994).

Tversky, A., Wakker, P. (1995). Risk attitudes and decision weights. Econometrica, 63, 1255-1280.

Von Neumann J. Morgenstern O. (1944). Theory of Games and Economic Behavior, Princeton University Press, Princeton (N.J.).

Von Winterfeldt, D. (1997). Empirical tests of Luce's rank- and sign-dependent utility theory. In A. A. J. Marley (Eds.), Choice, decision, and measurement: Essays in honor of R. Duncan Luce (pp. 25-44). Mahwah, NJ: Erlbaum.

Wakker, P. (1994). Separating marginal utility and probabilistic risk aversion. Theory and decision, 36, 1-44.

Wakker, P. (1996). The sure-thing principle and the comonotonic sure-thing principle: An axiomatic analysis. Journal of Mathematical Economics, 25, 213-227.

Wakker, P. (2001). Testing and characterizing properties of nonadditive measures through violations of the sure-thing principle. Econometrica, 69, 1039-1075.

Wakker, P., Erev, I., Weber, E. U. (1994). Comonotonic independence: The critical test between classical and rank-dependent utility theories. Journal of Risk and Uncertainty, 9, 195-230.

Weber, E. U., Kirsner, B. (1997). Reasons for rankdependent utility evaluation. Journal of Risk and Uncertainty, 14, 41-61.

Wenstein, N, (1980) unrealistic optimism about future life events Journal of personality and Social Psychology, 39

Wu, G. (1994). An empirical test of ordinal independence. Journal of Risk and Uncertainty, 9, 39-60.

Wu, G., Gonzalez, R. (1996). Curvature of the probability weighting function. Management Science, 42, 1676-1690. 
Wu, G., Gonzalez, R. (1998). Common consequence conditions in decision making under risk. Journal of Risk and Uncertainty, 16, 115-139.

Wu, G., Gonzalez, R. (1999). Nonlinear decision weights in choice under uncertainty. Management Science, 45, 74-85.

Wu, G., Markle, A. B. (2005). An empirical test of gain-loss separability in prospect theory. Working Manuscript, Available from George
Wu, University of Chicago, Graduate School of Business, 1101 E. 58 th Street, Chicago, IL 60637.

Wu, G., Zhang, J., Abdelloui, M. (2005). Testing prospect theories using probability tradeoff consistency. Journal of Risk and Uncertainty, 30, 107-131.

Yaari, M. E. (1987). The dual theory of choice under risk. Econometrica, 55, 95-115. 